\title{
An algorithm for a common minimum-norm zero of a finite family of monotone mappings in Banach spaces
}

\author{
Habtu Zegeye ${ }^{1}$ and Naseer Shahzad ${ }^{2 *}$
}

\section{"Correspondence:}

nshahzad@kau.edu.sa

${ }^{2}$ Department of Mathematics, King

Abdulaziz University, P.O. Box 80203

Jeddah, 21589, Saudi Arabia

Full list of author information is

available at the end of the article

\begin{abstract}
We introduce an iterative process which converges strongly to a common minimum-norm point of solutions of a finite family of monotone mappings in Banach spaces. Our theorems improve and unify most of the results that have been proved for this important class of nonlinear mappings.

MSC: 47H05; 47H09; 47H10;47J05; 47J25
\end{abstract}

Keywords: accretive mappings; maximal monotone mappings; proximal point algorithm; resolvent mappings

\section{Introduction}

In many problems, it is quite often to seek a particular solution of the minimum-norm solution of a given nonlinear problem. In an abstract way, we may formulate such problems as finding a point $x^{*}$ with the property

$$
x^{*} \in C \quad \text { such that } \quad\left\|x^{*}\right\|=\min _{x \in C}\{\|x\|\}
$$

where $C$ is a nonempty closed convex subset of a real Hilbert space $H$. In other words, $x^{*}$ is the (nearest point or metric) projection of the origin onto $C$,

$$
x^{*}=P_{C}(0),
$$

where $P_{C}$ is the metric (or nearest point) projection from $H$ onto $C$. For instance, the split feasibility problem (SFP), introduced in $[1,2]$, is to find a point

$$
x^{*} \in C \text { such that } A x^{*} \in Q \text {, }
$$

where $C$ and $Q$ are closed convex subsets of Hilbert spaces $H_{1}$ and $H_{2}$, respectively, and $A$ is a linear bounded operator from $H_{1}$ to $H_{2}$. We note that problem (1.3) can be extended to a problem of finding

$$
x \in D(A) \cap D(B) \quad \text { such that } \quad x \in A^{-1}(0) \cap B^{-1}(0),
$$

where $A: D(A) \rightarrow E^{*}$ and $B: D(B) \rightarrow E^{*}$ are monotone mappings on a subset of a Banach space $E$. The problem has been addressed by many authors in view of the applica-

@2013Zegeye and Shahzad; licensee Springer. This is an Open Access article distributed under the terms of the Creative Commons Attribution License (http://creativecommons.org/licenses/by/2.0), which permits unrestricted use, distribution, and reproduction in any medium, provided the original work is properly cited. 
tions in image recovery and signal processing; see, for example, [3-5] and the references therein.

A mapping $A: C \rightarrow E^{*}$ is said to be monotone if for each $x, y \in C$, the following inequality holds:

$$
\langle x-y, A x-A y\rangle \geq 0,
$$

where $C$ is a nonempty subset of a real Banach space $E$ with $E^{*}$ as its dual. $A$ is said to be maximal monotone if its graph is not properly contained in the graph of any other monotone mapping. A mapping $A: C \rightarrow E^{*}$ is said to be $\gamma$-inverse strongly monotone if there exists a positive real number $\gamma$ such that

$$
\langle x-y, A x-A y\rangle \geq \gamma\|A x-A y\|^{2} \quad \text { for all } x, y \in C,
$$

and it is called strongly monotone if there exists $k>0$ such that

$$
\langle x-y, A x-A y\rangle \geq k\|x-y\|^{2} \quad \text { for all } x, y \in C .
$$

An operator $A: C \rightarrow E$ is called accretive if there exists $j(x-y) \in J(x-y)$ such that

$$
\langle A x-A y, j(x-y)| \geq 0 \quad \text { for all } x, y \in C,
$$

where $J$ is the normalized duality mapping from $E$ into $2^{E^{*}}$ defined for each $x \in E$ by

$$
J x:=\left\{f^{*} \in E^{*}:\left\langle x, f^{*}\right\rangle=\|x\|^{2}=\left\|f^{*}\right\|^{2}\right\} .
$$

It is well known that $E$ is smooth if and only if $J$ is single-valued, and if $E$ is uniformly smooth, then $J$ is uniformly continuous on bounded subsets of $E$ (see [6]). $A$ is called $m$ accretive if it is accretive and $R(I+r A)$, the range of $(I+r A)$, is $E$ for all $r>0$; and an accretive mapping $A$ is said to satisfy range condition if

$$
D(A) \subseteq C \subseteq \bigcap_{r>0} R(I+r A)
$$

for some nonempty closed convex subset $C$ of a real Banach space $H$.

Clearly, the class of monotone mappings includes the class of strongly monotone and the class of $\gamma$-inverse strongly monotone mappings. However, we observe that accretive mappings and monotone mappings have different natures in Banach spaces more general than Hilbert spaces.

When $A$ and $B$ are maximal monotone mappings in Hilbert spaces, Bauschke et al. [7] proved that sequences generated from the method of alternating resolvents given by

$$
\begin{cases}x_{2 n+1}=J_{\beta}^{A}\left(x_{2 n}\right), & n \geq 0, \\ x_{2 n}=J_{\mu}^{B}\left(x_{2 n-1}\right), & n \geq 0,\end{cases}
$$

where $J_{\mu}^{A}:=(I+\mu A)^{-1}$ is the resolvent of $A$, converge weakly to a point of $A^{-1}(0) \cap B^{-1}(0)$ provided that $A^{-1}(0) \cap B^{-1}(0)$ is nonempty. Note that strong convergence of these methods fails in general (see a counter example by Hundal [8]). 
With regard to a finite family of $m$-accretive mappings, Zegeye and Shahzad [9] proved that under appropriate conditions, an iterative process of Halpern type defined by

$$
x_{n+1}=\alpha_{n} u+\left(1-\alpha_{n}\right) S_{r_{n}} x_{n}, \quad n \geq 0,
$$

where $\alpha_{n} \in(0,1)$ for all $n \geq 0, u, x_{0} \in H, S_{r}:=a_{0} I+a_{1} J_{r}^{1}+a_{2} J_{r}^{2}+\cdots+a_{N} J_{r}^{N}$ with $J_{r}^{i}=$ $\left(I+r A_{i}\right)^{-1}$ for $a_{i} \in(0,1), i=0,1, \ldots, N$, and $\sum_{i=1} a_{i}^{N}=1$, converges strongly to a point in $\bigcap_{i=1}^{N} A^{-1}(0)$ nearest to $u$, where $\left\{A_{i}: i=1,2, \ldots, N\right\}$ is the set of a finite family of $m$-accretive mappings in a strictly convex and reflexive (real) Banach space $E$ which has a uniformly Gâteaux differentiable norm.

In 2009, $\mathrm{Hu}$ and Liu [10] also proved that under appropriate conditions, an iterative process of Halpern type defined by

$$
x_{n+1}=\alpha_{n} u+\delta_{n} x_{n}+\gamma_{n} S_{r_{n}} x_{n}, \quad n \geq 0,
$$

where $\alpha_{n}, \delta_{n}, \gamma_{n} \in(0,1)$ with $\alpha_{n}+\delta_{n}+\gamma_{n}=1$, for all $n \geq 0, u=x_{0} \in H, S_{r_{n}}:=a_{0} I+a_{1} J_{r_{n}}^{1}+$ $a_{2} J_{r_{n}}^{2}+\cdots+a_{N} J_{r_{n}}^{N}$ with $J_{r}^{i}=\left(I+r A_{i}\right)^{-1}$, for $a_{i} \in(0,1), i=0,1, \ldots, N$, and $\sum_{i=1} a_{i}^{N}=1$, and $\left\{r_{n}\right\} \subset(0, \infty)$, for $A_{i}, i=1,2, \ldots, N$, accretive mappings satisfying range condition (1.9), converges strongly to a point in $\bigcap_{i=1}^{N} A^{-1}(0)$ nearest to $u$ in a strictly convex and reflexive (real) Banach space $E$ which has a uniformly Gâteaux differentiable norm.

A natural question arises whether we can have the results of Zegeye and Shahzad [9] and $H u$ and Liu [10] for the class of monotone mappings or not, in Banach spaces more general than Hilbert spaces?

Let $C$ be a nonempty, closed, and convex subset of a smooth and uniformly convex real Banach space $E$. Let $A_{i}: C \rightarrow E^{*}$ for $i=1,2, \ldots, N$ be continuous monotone mappings satisfying range condition (2.1) with $F:=\bigcap_{i=1}^{N} A_{i}^{-1}(0) \neq \emptyset$.

It is our purpose in this paper to introduce an iterative scheme (see (3.1)) which converges strongly to the common minimum-norm zero of the family $\left\{A_{i}, i=1,2, \ldots, N\right\}$. Our theorems improve and unify most of the results that have been proved for this important class of nonlinear mappings.

\section{Preliminaries}

Let $E$ be a normed linear space with $\operatorname{dim} E \geq 2$. The modulus of smoothness of $E$ is the function $\rho_{E}:[0, \infty) \rightarrow[0, \infty)$ defined by

$$
\rho_{E}(\tau):=\sup \left\{\frac{\|x+y\|+\|x-y\|}{2}-1:\|x\|=1 ;\|y\|=\tau\right\} .
$$

The space $E$ is said to be smooth if $\rho_{E}(\tau)>0, \forall \tau>0$, and $E$ is called uniformly smooth if and only if $\lim _{t \rightarrow 0^{+}} \frac{\rho_{E}(t)}{t}=0$.

The modulus of convexity of $E$ is the function $\delta_{E}:(0,2] \rightarrow[0,1]$ defined by

$$
\delta_{E}(\epsilon):=\inf \left\{1-\left\|\frac{x+y}{2}\right\|:\|x\|=\|y\|=1 ; \epsilon=\|x-y\|\right\} .
$$

$E$ is called uniformly convex if and only if $\delta_{E}(\epsilon)>0$ for every $\epsilon \in(0,2]$. 
Let $C$ be a nonempty, closed, and convex subset of a smooth, strictly convex, and reflexive Banach space $E$ with dual $E^{*}$. A monotone mapping $A$ is said to satisfy range condition if we have that

$$
D(A) \subseteq C \subseteq \bigcap_{r>0} J^{-1} R(J+r A)
$$

for some nonempty closed convex subset $C$ of a smooth, strictly convex, and reflexive Banach space $E$. In the sequel, the resolvent of a monotone mapping $A: C \rightarrow E^{*}$ shall be denoted by $Q_{r}^{A}:=(J+r A)^{-1} J$ for $r>0$. We know the following lemma.

Lemma 2.1 [11] Let $E$ be a smooth and strictly convex Banach space, $C$ be a nonempty, closed, and convex subset of $E$, and $A \subset E \times E^{*}$ be a monotone mapping satisfying (2.1). Let $Q_{r_{n}}^{A}$ be the resolvent of $A$ for $\left\{r_{n}\right\} \subset(0, \infty)$ such that $\lim _{n \rightarrow \infty} r_{n}=\infty$. If $\left\{x_{n}\right\}$ is a bounded sequence of $C$ such that $Q_{r_{n}}^{A} x_{n} \rightarrow z$, then $z \in A^{-1}(0)$.

Let $E$ be a smooth Banach space with dual $E^{*}$. Let the Lyapunov function $\phi: E \times E \rightarrow \mathbb{R}$, introduced by Alber [12], be defined by

$$
\phi(y, x)=\|y\|^{2}-2\langle y, J x\rangle+\|x\|^{2} \quad \text { for } x, y \in E,
$$

where $J$ is the normalized duality mapping. If $E=H$, a Hilbert space, then the duality mapping becomes the identity map on $H$. We observe that in a Hilbert space $H,(2.2)$ reduces to $\phi(x, y)=\|x-y\|^{2}$ for $x, y \in H$.

In the sequel, we shall make use of the following lemmas.

Lemma 2.2 [13] Let E be a smooth and strictly convex Banach space, and C be a nonempty, closed, and convex subset of $E$. Let $A \subset E \times E^{*}$ be a monotone mapping satisfying (2.1), $A^{-1}(0)$ be nonempty and $Q_{r}^{A}$ be the resolvent of $A$ for some $r>0$. Then, for each $r>0$, we have that

$$
\phi\left(p, Q_{r}^{A} x\right)+\phi\left(Q_{r}^{A} x, x\right) \leq \phi(p, x)
$$

for all $p \in A^{-1}(0)$ and $x \in C$.

Lemma 2.3 [14] Let E be a smooth and strictly convex Banach space, $C$ be a nonempty, closed, and convex subset of $E$, and $T$ be a mapping from $C$ into itself such that $F(T)$ is nonempty and $\phi(p, T x) \leq \phi(p, x)$ for all $p \in F(T)$ and $x \in C$. Then $F(T)$ is closed and convex.

Lemma 2.4 [15] Let E be a real smooth and uniformly convex Banach space, and let $\left\{x_{n}\right\}$ and $\left\{y_{n}\right\}$ be two sequences of E. If either $\left\{x_{n}\right\}$ or $\left\{y_{n}\right\}$ is bounded and $\phi\left(x_{n}, y_{n}\right) \rightarrow 0$ as $n \rightarrow \infty$, then $x_{n}-y_{n} \rightarrow 0$ as $n \rightarrow \infty$.

We make use of the function $V: E \times E^{*} \rightarrow \mathbb{R}$ defined by

$$
V\left(x, x^{*}\right)=\|x\|^{2}-2\left\langle x, x^{*}\right\rangle+\left\|x^{*}\right\|^{2} \quad \text { for all } x \in E \text { and } x^{*} \in E,
$$

studied by Alber [12]. That is, $V\left(x, x^{*}\right)=\phi\left(x, J^{-1} x^{*}\right)$ for all $x \in E$ and $x^{*} \in E^{*}$. 
Lemma 2.5 [12] Let E be a reflexive, strictly convex, and smooth Banach space with $E^{*}$ as its dual. Then

$$
V\left(x, x^{*}\right)+2\left(J^{-1} x^{*}-x, y^{*}\right) \leq V\left(x, x^{*}+y^{*}\right)
$$

for all $x \in E$ and $x^{*}, y^{*} \in E^{*}$.

Let $E$ be a reflexive, strictly convex, and smooth Banach space, and let $C$ be a nonempty, closed, and convex subset of $E$. The generalized projection mapping, introduced by Alber [12], is a mapping $\Pi_{C}: E \rightarrow C$ that assigns an arbitrary point $x \in E$ to the minimizer, $\bar{x}$, of $\phi(\cdot, x)$ over $C$, that is, $\Pi_{C} x=\bar{x}$, where $\bar{x}$ is the solution to the minimization problem

$$
\phi(\bar{x}, x)=\min \{\phi(y, x), y \in C\} .
$$

Lemma 2.6 [12] Let $C$ be a nonempty, closed, and convex subset of a real reflexive, strictly convex, and smooth Banach space $E$, and let $x \in E$. Then, $\forall y \in C$,

$$
\phi\left(y, \Pi_{C} x\right)+\phi\left(\Pi_{C} x, x\right) \leq \phi(y, x) .
$$

Lemma 2.7 [12] Let $C$ be a convex subset of a real smooth Banach space E. Let $x \in E$. Then $x_{0}=\Pi_{C} x$ if and only if

$$
\left\langle z-x_{0}, J x-J x_{0}\right\rangle \leq 0, \quad \forall z \in C
$$

Lemma 2.8 [16] Let E be a uniformly convex Banach space and $B_{R}(0)$ be a closed ball of $E$. Then there exists a continuous strictly increasing convex function $g:[0, \infty) \rightarrow[0, \infty)$ with $g(0)=0$ such that

$$
\left\|\alpha_{0} x_{0}+\alpha_{1} x_{1}+\cdots+\alpha_{N} x_{N}\right\|^{2} \leq \sum_{i=0}^{N} \alpha_{i}\left\|x_{i}\right\|^{2}-\alpha_{i} \alpha_{j} g\left(\left\|x_{i}-x_{j}\right\|\right)
$$

for $\alpha_{i} \in(0,1)$ such that $\sum_{i=0}^{N} \alpha_{i}=1$ and $x_{i} \in B_{R}(0):=\{x \in E:\|x\| \leq R\}$ for some $R>0$.

Lemma 2.9 [17] Let $\left\{a_{n}\right\}$ be a sequence of nonnegative real numbers satisfying the following relation:

$$
a_{n+1} \leq\left(1-\beta_{n}\right) a_{n}+\beta_{n} \delta_{n}, \quad \forall n \geq n_{0},
$$

where $\left\{\beta_{n}\right\} \subset(0,1)$ and $\left\{\delta_{n}\right\} \subset R$ satisfy the following conditions: $\lim _{n \rightarrow \infty} \beta_{n}=0, \sum_{n=1}^{\infty} \beta_{n}=$ $\infty$, and $\lim \sup _{n \rightarrow \infty} \delta_{n} \leq 0$. Then $\lim _{n \rightarrow \infty} a_{n}=0$.

Lemma 2.10 [18] Let $\left\{a_{n}\right\}$ be sequences of real numbers such that there exists a subsequence $\left\{n_{i}\right\}$ of $\{n\}$ such that $a_{n_{i}}<a_{n_{i}+1}$ for all $i \in \mathbb{N}$. Then there exists a nondecreasing sequence $\left\{m_{k}\right\} \subset \mathbb{N}$ such that $m_{k} \rightarrow \infty$, and the following properties are satisfied by all (sufficiently large) numbers $k \in \mathbb{N}$ :

$$
a_{m_{k}} \leq a_{m_{k}+1} \text { and } a_{k} \leq a_{m_{k}+1} \text {. }
$$


In fact, $m_{k}$ is the largest number $n$ in the set $\{1,2, \ldots, k\}$ such that the condition $a_{n} \leq a_{n+1}$ holds.

\section{Main result}

We now prove the following theorem.

Theorem 3.1 Let $C$ be a nonempty, closed, and convex subset of a smooth and uniformly convex real Banach space $E$. Let $A_{i}: C \rightarrow E^{*}$, for $i=1,2, \ldots, N$, be continuous monotone mappings satisfying (2.1). Assume that $\mathcal{F}:=\bigcap_{i=1}^{N} A_{i}^{-1}(0)$ is nonempty. Let $\left\{x_{n}\right\}$ be a sequence generated by

$$
\left\{\begin{array}{l}
x_{0} \in C, \quad \text { chosen arbitrarily, } \\
y_{n}=\Pi_{C}\left[\left(1-\alpha_{n}\right) x_{n}\right], \\
x_{n+1}=J^{-1}\left(\beta_{0} J y_{n}+\sum_{i=1}^{N} \beta_{j} J Q_{r_{n}}^{A_{i}} y_{n}\right), \quad \forall n \geq 0,
\end{array}\right.
$$

where $\alpha_{n} \in(0,1),\left\{\beta_{i}\right\}_{i=1}^{N} \subset[c, d] \subset(0,1)$ and $\left\{r_{n}\right\} \subset(0, \infty)$ satisfy the following conditions: $\lim _{n \rightarrow \infty} \alpha_{n}=0, \sum_{n=1}^{\infty} \alpha_{n}=\infty, \sum_{i=0}^{N} \beta_{i}=1$, and $\lim _{n \rightarrow \infty} r_{n}=\infty$. Then $\left\{x_{n}\right\}$ converges strongly to the minimum-norm point of $\mathcal{F}$.

Proof From Lemmas 2.2 and 2.3 we get that $A_{i}^{-1}(0)$ is closed and convex. Thus, $\Pi_{\mathcal{F}}(0)$ is well defined. Let $p=\Pi_{\mathcal{F}}(0)$. Then from (3.1), Lemma 2.6 and the property of $\phi$, we get that

$$
\begin{aligned}
\phi\left(p, y_{n}\right)= & \phi\left(p, \Pi_{C}\left(1-\alpha_{n}\right) x_{n}\right) \leq \phi\left(p,\left(1-\alpha_{n}\right) x_{n}\right) \\
= & \phi\left(p, J^{-1}\left(\alpha_{n} J 0+\left(1-\alpha_{n}\right) J x_{n}\right)\right) \\
= & \|p\|^{2}-2\left\langle p, \alpha_{n} J 0+\left(1-\alpha_{n}\right) J x_{n}\right\rangle+\left\|\alpha_{n} J 0+\left(1-\alpha_{n}\right) J x_{n}\right\|^{2} \\
\leq & \|p\|^{2}-2 \alpha_{n}\langle p, J 0\rangle-2\left(1-\alpha_{n}\right)\left\langle p, J x_{n}\right\rangle \\
& +\alpha_{n}\|J 0\|^{2}+\left(1-\alpha_{n}\right)\left\|J x_{n}\right\|^{2} \\
= & \alpha_{n} \phi(p, 0)+\left(1-\alpha_{n}\right) \phi\left(p, x_{n}\right) .
\end{aligned}
$$

Moreover, from (3.1), Lemma 2.8, Lemma 2.2 and (3.2), we get that

$$
\begin{aligned}
\phi\left(p, x_{n+1}\right)= & \phi\left(p, J^{-1}\left(\beta_{0} J y_{n}+\sum_{i=1}^{N} \beta_{j} J Q_{r_{n}}^{A_{i}} y_{n}\right)\right) \\
= & \|p\|^{2}-2\left\langle p, \beta_{0} J y_{n}+\sum_{i=1}^{N} \beta_{i} J Q_{r_{n}}^{A_{i}} y_{n}\right\rangle+\left\|\beta_{0} J y_{n}+\sum_{i=1}^{N} \beta_{i} J Q_{r_{n}}^{A_{i}} y_{n}\right\|^{2} \\
\leq & \|p\|^{2}-2 \beta_{0}\left\langle p, J y_{n}\right\rangle-2 \sum_{i=1}^{N} \beta_{i}\left\langle p, J Q_{r_{n}}^{A_{i}} y_{n}\right\rangle \\
& +\beta_{0}\left\|y_{n}\right\|^{2}+\sum_{i=1}^{N} \beta_{i}\left\|Q_{r_{n}}^{A_{i}} y_{n}\right\|^{2}-\beta_{0} \beta_{i} g\left(\left\|J y_{n}-J Q_{r_{n}}^{A_{i}} y_{n}\right\|\right) \\
= & \beta_{0} \phi\left(p, y_{n}\right)+\sum_{i=1}^{N} \beta_{i} \phi\left(p, Q_{r_{n}}^{A_{i}} y_{n}\right)-\beta_{0} \beta_{i} g\left(\left\|J y_{n}-J Q_{r_{n}}^{A_{i}} y_{n}\right\|\right)
\end{aligned}
$$




$$
\begin{aligned}
& \leq \beta_{0} \phi\left(p, y_{n}\right)+\left(1-\beta_{0}\right) \phi\left(p, y_{n}\right)-\beta_{0} \beta_{i} g\left(\left\|J y_{n}-J Q_{r_{n}}^{A_{i}} y_{n}\right\|\right) \\
& \leq \phi\left(p, y_{n}\right)-\beta_{0} \beta_{i} g\left(\left\|J y_{n}-J Q_{r_{n}}^{A_{i}} y_{n}\right\|\right) \leq \phi\left(p, y_{n}\right) \\
& \leq \alpha_{n} \phi(p, 0)+\left(1-\alpha_{n}\right) \phi\left(p, x_{n}\right)
\end{aligned}
$$

for each $i \in\{1,2, \ldots, N\}$. Thus, by induction,

$$
\phi\left(p, x_{n+1}\right) \leq \max \left\{\phi(p, 0), \phi\left(p, x_{0}\right)\right\}, \quad \forall n \geq 0,
$$

which implies that $\left\{x_{n}\right\}$ and hence $\left\{y_{n}\right\}$ are bounded. Now let $z_{n}=\left(1-\alpha_{n}\right) x_{n}$. Then we note that $y_{n}=\Pi_{C} z_{n}$. Using Lemma 2.6, Lemma 2.5 and the property of $\phi$, we obtain that

$$
\begin{aligned}
\phi\left(p, y_{n}\right) & \leq \phi\left(p, z_{n}\right)=V\left(p, J z_{n}\right) \\
& \leq V\left(p, J z_{n}-\alpha_{n}(J 0-J p)\right)-2\left\langle z_{n}-p,-\alpha_{n}(J 0-J p)\right\rangle \\
& =\phi\left(p, J^{-1}\left(\alpha_{n} J p+\left(1-\alpha_{n}\right) J x_{n}\right)\right)-2 \alpha_{n}\left\langle z_{n}-p, J p\right\rangle \\
& \leq \alpha_{n} \phi(p, p)+\left(1-\alpha_{n}\right) \phi\left(p, x_{n}\right)-2 \alpha_{n}\left\langle z_{n}-p, J p\right\rangle \\
& =\left(1-\alpha_{n}\right) \phi\left(p, x_{n}\right)-2 \alpha_{n}\left\langle z_{n}-p, J p\right\rangle \\
& \leq\left(1-\alpha_{n}\right) \phi\left(p, x_{n}\right)-2 \alpha_{n}\left\langle z_{n}-p, J p\right\rangle .
\end{aligned}
$$

Furthermore, from (3.3) and (3.5) we have that

$$
\begin{aligned}
\phi\left(p, x_{n+1}\right) \leq & \left(1-\alpha_{n}\right) \phi\left(p, x_{n}\right)-2 \alpha_{n}\left\langle z_{n}-p, J p\right\rangle \\
& -\beta_{0} \beta_{i} g\left(\left\|J y_{n}-J Q_{r_{n}}^{A_{i}} y_{n}\right\|\right) \\
\leq & \left(1-\alpha_{n}\right) \phi\left(p, x_{n}\right)-2 \alpha_{n}\left\langle z_{n}-p, J p\right\rangle .
\end{aligned}
$$

Now, following the method of proof of Lemma 3.2 of Maingé [18], we consider two cases as follows.

Case 1. Suppose that there exists $n_{0} \in \mathbb{N}$ such that $\left\{\phi\left(p, x_{n}\right)\right\}$ is nonincreasing for all $n \geq n_{0}$. In this situation, $\left\{\phi\left(p, x_{n}\right)\right\}$ is convergent. Then from (3.6) we have that

$$
\beta_{0} \beta_{i} g\left(\left\|J y_{n}-J Q_{r_{n}}^{A_{i}} y_{n}\right\|\right) \rightarrow 0
$$

which implies, by the property of $g$, that

$$
J y_{n}-J Q_{r_{n}}^{A_{i}} y_{n} \rightarrow 0 \quad \text { as } n \rightarrow \infty
$$

and hence, since $J^{-1}$ is uniformly continuous on bounded sets, we obtain that

$$
y_{n}-Q_{r_{n}}^{A_{i}} y_{n} \rightarrow 0 \quad \text { as } n \rightarrow \infty
$$

for each $i \in\{1,2, \ldots, N\}$. 
Furthermore, Lemma 2.6, the property of $\phi$ and the fact that $\alpha_{n} \rightarrow 0$, as $n \rightarrow \infty$, imply that

$$
\begin{aligned}
\phi\left(x_{n}, y_{n}\right) & =\phi\left(x_{n}, \Pi_{C} z_{n}\right) \\
& \leq \phi\left(x_{n}, z_{n}\right) \\
& =\phi\left(x_{n}, J^{-1}\left(\alpha_{n} J 0+\left(1-\alpha_{n}\right) J x_{n}\right)\right) \\
& \leq \alpha_{n} \phi\left(x_{n}, 0\right)+\left(1-\alpha_{n}\right) \phi\left(x_{n}, x_{n}\right) \\
& \leq \alpha_{n} \phi\left(x_{n}, 0\right)+\left(1-\alpha_{n}\right) \phi\left(x_{n}, x_{n}\right) \rightarrow 0 \quad \text { as } n \rightarrow \infty,
\end{aligned}
$$

and hence from Lemma 2.4 we get that

$$
x_{n}-y_{n} \rightarrow 0, \quad x_{n}-z_{n} \rightarrow 0 \quad \text { as } n \rightarrow \infty .
$$

Since $\left\{z_{n}\right\}$ is bounded and $E$ is reflexive, we choose a subsequence $\left\{z_{n_{i}}\right\}$ of $\left\{z_{n}\right\}$ such that $z_{n_{i}} \rightarrow z$ and $\lim \sup _{n \rightarrow \infty}\left\langle z_{n}-p, J p\right\rangle=\lim _{i \rightarrow \infty}\left\langle z_{n_{i}}-p, J p\right\rangle$. Then from (3.12) we get that

$$
y_{n_{i}} \rightarrow z \quad \text { as } i \rightarrow \infty .
$$

Thus, from (3.10) and Lemma 2.1, we obtain that $z \in A_{i}^{-1}(0)$ for each $i \in\{1,2, \ldots, N\}$ and hence $z \in \bigcap_{i=1}^{N} A_{i}^{-1}(0)$.

Therefore, by Lemma 2.7 , we immediately obtain that $\lim _{\sup _{n \rightarrow \infty}}\left\langle z_{n}-p, J p\right\rangle=$ $\lim _{i \rightarrow \infty}\left\langle z_{n_{i}}-p, J p\right\rangle=\langle z-p, J p\rangle \geq 0$. It follows from Lemma 2.9 and (3.7) that $\phi\left(p, x_{n}\right) \rightarrow 0$ as $n \rightarrow \infty$. Consequently, from Lemma 2.4 we obtain that $x_{n} \rightarrow p$.

Case 2. Suppose that there exists a subsequence $\left\{n_{i}\right\}$ of $\{n\}$ such that

$$
\phi\left(p, x_{n_{i}}\right)<\phi\left(p, x_{n_{i}+1}\right)
$$

for all $i \in \mathbb{N}$. Then, by Lemma 2.10 , there exists a nondecreasing sequence $\left\{m_{k}\right\} \subset \mathbb{N}$ such that $m_{k} \rightarrow \infty, \phi\left(p, x_{m_{k}}\right) \leq \phi\left(p, x_{m_{k}+1}\right)$, and $\phi\left(p, x_{k}\right) \leq \phi\left(p, x_{m_{k}+1}\right)$ for all $k \in \mathbb{N}$. Then, from (3.6) and the fact that $\alpha_{n} \rightarrow 0$, we obtain that

$$
g\left(\left\|J y_{m_{k}}-J Q_{r_{m_{k}}}^{A_{i}} y_{m_{k}}\right\|\right) \rightarrow 0 \quad \text { as } k \rightarrow \infty
$$

for each $i \in\{1,2, \ldots, N\}$. Thus, following the method of proof of Case 1, we obtain that $y_{m_{k}}-Q_{r_{m_{k}}}^{A_{i}} y_{m_{k}} \rightarrow 0, x_{m_{k}}-y_{m_{k}} \rightarrow 0, x_{m_{k}}-z_{m_{k}} \rightarrow 0$ as $k \rightarrow \infty$, and hence we obtain that

$$
\limsup _{k \rightarrow \infty}\left\langle z_{m_{k}}-p, J p\right\rangle \geq 0 .
$$

Then from (3.7) we have that

$$
\phi\left(p, x_{m_{k}+1}\right) \leq\left(1-\alpha_{m_{k}}\right) \phi\left(p, x_{m_{k}}\right)-2 \alpha_{m_{k}}\left\langle z_{m_{k}}-p, J p\right\rangle .
$$

Now, since $\phi\left(p, x_{m_{k}}\right) \leq \phi\left(p, x_{m_{k}+1}\right)$, inequality (3.15) implies that

$$
\begin{aligned}
\alpha_{m_{k}} \phi\left(p, x_{m_{k}}\right) & \leq \phi\left(p, x_{m_{k}}\right)-\phi\left(p, x_{m_{k}+1}\right)-2 \alpha_{m_{k}}\left\langle z_{m_{k}}-p, J p\right\rangle \\
& \leq-2 \alpha_{m_{k}}\left\langle z_{m_{k}}-p, J p\right\rangle .
\end{aligned}
$$


In particular, since $\alpha_{m_{k}}>0$, we get

$$
\phi\left(p, x_{m_{k}}\right) \leq-2\left\langle z_{m_{k}}-p, J p\right\rangle
$$

Then from (3.14) we obtain $\phi\left(p, x_{m_{k}}\right) \rightarrow 0$ as $k \rightarrow \infty$. This together with (3.15) gives $\phi\left(p, x_{m_{k}+1}\right) \rightarrow 0$ as $k \rightarrow \infty$. But $\phi\left(p, x_{k}\right) \leq \phi\left(p, x_{m_{k}+1}\right)$ for all $k \in \mathbb{N}$, thus we obtain that $x_{k} \rightarrow p$. Therefore, from the above two cases, we can conclude that $\left\{x_{n}\right\}$ converges strongly to $p$, which is the common minimum-norm zero of the family $\left\{A_{i}, i=1,2, \ldots, N\right\}$, and the proof is complete.

We would like to mention that the method of proof of Theorem 3.1 provides the following theorem.

Theorem 3.2 Let $C$ be a nonempty, closed, and convex subset of a smooth and uniformly convex real Banach space E. Let $A_{i}: C \rightarrow E^{*}$, for $i=1,2, \ldots, N$, be continuous monotone mappings satisfying (2.1). Assume that $\mathcal{F}:=\bigcap_{i=1}^{N} A_{i}^{-1}(0)$ is nonempty. Let $\left\{x_{n}\right\}$ be a sequence generated by

$$
\left\{\begin{array}{l}
u=x_{0} \in C, \quad \text { chosen arbitrarily, } \\
y_{n}=\Pi_{C} J^{-1}\left(\alpha_{n} J u+\left(1-\alpha_{n}\right) J x_{n}\right), \\
x_{n+1}=J^{-1}\left(\beta_{0} J y_{n}+\sum_{i=1}^{N} \beta_{i} J Q_{r_{n}}^{A_{i}} y_{n}\right), \quad \forall n \geq 0,
\end{array}\right.
$$

where $\alpha_{n} \in(0,1),\left\{\beta_{i}\right\}_{i=1}^{N} \subset[c, d] \subset(0,1)$, and $\left\{r_{n}\right\} \subset(0, \infty)$ satisfy $\lim _{n \rightarrow \infty} \alpha_{n}=0, \sum_{n=1}^{\infty} \alpha_{n}=$ $\infty, \sum_{i=0}^{N} \beta_{i}=1$, and $\lim _{n \rightarrow \infty} r_{n}=\infty$. Then $\left\{x_{n}\right\}$ converges strongly to $\Pi_{\mathcal{F}}(u)$.

If in Theorem 3.1, $N=1$, then we get the following corollary.

Corollary 3.3 Let $C$ be a nonempty, closed, and convex subset of a smooth and uniformly convex real Banach space E. Let $A: C \rightarrow E^{*}$ be a continuous monotone mapping satisfying (2.1). Assume that $A^{-1}(0)$ is nonempty. Let $\left\{x_{n}\right\}$ be a sequence generated by

$$
\left\{\begin{array}{l}
x_{0} \in C, \quad \text { chosen arbitrarily, } \\
y_{n}=\Pi_{C}\left[\left(1-\alpha_{n}\right) x_{n}\right], \\
x_{n+1}=J^{-1}\left(\beta J y_{n}+(1-\beta) J Q_{r_{n}}^{A} y_{n}\right), \quad \forall n \geq 0,
\end{array}\right.
$$

where $\alpha_{n} \in(0,1), \beta \in(0,1)$, and $\left\{r_{n}\right\} \subset(0, \infty)$ satisfy $\lim _{n \rightarrow \infty} \alpha_{n}=0, \sum_{n=1}^{\infty} \alpha_{n}=\infty$, and $\lim _{n \rightarrow \infty} r_{n}=\infty$. Then $\left\{x_{n}\right\}$ converges strongly to the minimum-norm element of $A^{-1}(0)$.

We remark that if $A$ is a maximal monotone mapping, then $A^{-1}(0)$ is closed and convex (see [6] for more details). The following lemma is well known.

Lemma 3.4 [19] Let E be a smooth, strictly convex, and reflexive Banach space, let $C$ be a nonempty closed convex subset of $E$, and let $A \subset E \times E^{*}$ be a monotone mapping. Then $A$ is maximal if and only if $R(J+r A)=E^{*}$ for all $r>0$.

We note from the above lemma that if $A$ is maximal, then it satisfies condition (2.1) and hence we have the following corollary. 
Corollary 3.5 Let $C$ be a nonempty, closed, and convex subset of a smooth and uniformly convex real Banach space $E$. Let $A_{i}: C \rightarrow E^{*}, i=1,2, \ldots, N$, be maximal monotone mappings. Assume that $\mathcal{F}:=\bigcap_{i=1}^{N} A_{i}^{-1}(0)$ is nonempty. Let $\left\{x_{n}\right\}$ be a sequence generated by

$$
\left\{\begin{array}{l}
x_{0} \in C, \quad \text { chosen arbitrarily, } \\
y_{n}=\Pi_{C}\left[\left(1-\alpha_{n}\right) x_{n}\right], \\
x_{n+1}=J^{-1}\left(\beta_{0} J y_{n}+\sum_{i=1}^{N} \beta_{i} J Q_{r_{n}}^{A_{i}} y_{n}\right), \quad \forall n \geq 0,
\end{array}\right.
$$

where $\alpha_{n} \in(0,1),\left\{\beta_{i}\right\}_{i=1}^{N} \subset[c, d] \subset(0,1)$ and $\left\{r_{n}\right\} \subset(0, \infty)$ satisfy $\lim _{n \rightarrow \infty} \alpha_{n}=0, \sum_{n=1}^{\infty} \alpha_{n}=$ $\infty, \sum_{i=0}^{N} \beta_{i}=1$ and $\lim _{n \rightarrow \infty} r_{n}=\infty$. Then $\left\{x_{n}\right\}$ converges strongly to the minimum-norm element of $\mathcal{F}$.

If in Corollary 3.5, $N=1$, then we get the following corollary.

Corollary 3.6 Let $C$ be a nonempty, closed and convex subset of a smooth and uniformly convex real Banach space E. Let $A: C \rightarrow E^{*}$ be a maximal monotone mapping. Assume that $A^{-1}(0)$ is nonempty. Let $\left\{x_{n}\right\}$ be a sequence generated by

$$
\left\{\begin{array}{l}
x_{0} \in C, \quad \text { chosen arbitrarily, } \\
y_{n}=\Pi_{C}\left[\left(1-\alpha_{n}\right) x_{n}\right], \\
x_{n+1}=J^{-1}\left(\beta J y_{n}+(1-\beta) J Q_{r_{n}}^{A} y_{n}\right), \quad \forall n \geq 0,
\end{array}\right.
$$

where $\alpha_{n} \in(0,1), \beta \in(0,1)$, and $\left\{r_{n}\right\} \subset(0, \infty)$ satisfy $\lim _{n \rightarrow \infty} \alpha_{n}=0, \sum_{n=1}^{\infty} \alpha_{n}=\infty$, and $\lim _{n \rightarrow \infty} r_{n}=\infty$. Then $\left\{x_{n}\right\}$ converges strongly to the minimum-norm element of $A^{-1}(0)$.

If $E=H$, a real Hilbert space, then $E$ is uniformly convex and smooth real Banach space. In this case, $J=I$, identity map on $H$, and $\Pi_{C}=P_{C}$, projection mapping from $H$ onto $C$. Furthermore, (2.1) reduces to (1.9). Thus, the following corollaries hold.

Corollary 3.7 Let C be a nonempty, closed, and convex subset of a real Hilbert space H. Let $A_{i}: C \rightarrow E^{*}$, for $i=1,2, \ldots, N$, be continuous monotone mappings satisfying (1.9). Assume that $\mathcal{F}:=\bigcap_{i=1}^{N} A_{i}^{-1}(0)$ is nonempty. Let $\left\{x_{n}\right\}$ be a sequence generated by

$$
\left\{\begin{array}{l}
x_{0} \in C, \quad \text { chosen arbitrarily, } \\
y_{n}=P_{C}\left[\left(1-\alpha_{n}\right) x_{n}\right], \\
x_{n+1}=\beta_{0} y_{n}+\sum_{i=1}^{N} \beta_{i} Q_{r_{n}}^{A_{i}} y_{n}, \quad \forall n \geq 0,
\end{array}\right.
$$

where $Q_{r}^{A}:=(I+r A)^{-1}, \alpha_{n} \in(0,1),\left\{\beta_{i}\right\}_{i=1}^{N} \subset[c, d] \subset(0,1)$, and $\left\{r_{n}\right\} \subset(0, \infty)$ satisfy $\lim _{n \rightarrow \infty} \alpha_{n}=0, \sum_{n=1}^{\infty} \alpha_{n}=\infty, \sum_{i=0}^{N} \beta_{i}=1$, and $\lim _{n \rightarrow \infty} r_{n}=\infty$. Then $\left\{x_{n}\right\}$ converges strongly to the minimum-norm element of $\mathcal{F}$.

Corollary 3.8 Let C be a nonempty, closed, and convex subset of a real Hilbertspace H. Let $A_{i}: C \rightarrow H, i=1,2, \ldots, N$, be maximal monotone mappings. Assume that $\mathcal{F}:=\bigcap_{i=1}^{N} A_{i}^{-1}(0)$ 
is nonempty. Let $\left\{x_{n}\right\}$ be a sequence generated by

$$
\left\{\begin{array}{l}
x_{0} \in C, \quad \text { chosen arbitrarily, } \\
y_{n}=P_{C}\left[\left(1-\alpha_{n}\right) x_{n}\right] \\
x_{n+1}=\beta_{0} y_{n}+\sum_{i=1}^{N} \beta_{i} Q_{r_{n}}^{A_{i}} y_{n}, \quad \forall n \geq 0
\end{array}\right.
$$

where $Q_{r}^{A}:=(I+r A)^{-1}, \alpha_{n} \in(0,1),\left\{\beta_{i}\right\}_{i=1}^{N} \subset[c, d] \subset(0,1)$, and $\left\{r_{n}\right\} \subset(0, \infty)$ satisfy $\lim _{n \rightarrow \infty} \alpha_{n}=0, \sum_{n=1}^{\infty} \alpha_{n}=\infty, \sum_{i=0}^{N} \beta_{i}=1$, and $\lim _{n \rightarrow \infty} r_{n}=\infty$. Then $\left\{x_{n}\right\}$ converges strongly to the minimum-norm element of $\mathcal{F}$.

\section{Application}

In this section, we study the problem of finding a minimizer of a continuously Fréchet differentiable convex functional which has minimum-norm in Banach spaces. The following is deduced from Corollary 3.6.

Theorem 4.1 Let E be a uniformly convex and uniformly smooth real Banach space. Let $f_{i}$ be a continuously Fréchet differentiable convex functional on $E$, and let $\nabla f_{i}$ be maximal monotone with $\mathcal{F}:=\bigcap_{i=1}^{N}\left(\nabla f_{i}\right)^{-1}(0) \neq \emptyset$, where $\left(\nabla f_{i}\right)^{-1}(0)=\left\{z \in E: f_{i}(z)=\min _{y \in E} f_{i}(y)\right\}$, for $i=1,2, \ldots, N$. Let $\left\{x_{n}\right\}$ be a sequence generated by

$$
\left\{\begin{array}{l}
x_{0} \in C, \quad \text { chosen arbitrarily, } \\
y_{n}=\Pi_{C}\left[\left(1-\alpha_{n}\right) x_{n}\right], \\
x_{n+1}=J^{-1}\left(\beta_{0} J y_{n}+\sum_{i=1}^{N} \beta_{i} J\left(J+r_{n} \nabla f_{i}\right)^{-1} J y_{n}\right), \quad \forall n \geq 0,
\end{array}\right.
$$

where $\alpha_{n} \in(0,1),\left\{\beta_{i}\right\}_{i=1}^{N} \subset[c, d] \subset(0,1)$, and $\left\{r_{n}\right\} \subset(0, \infty)$ satisfy $\lim _{n \rightarrow \infty} \alpha_{n}=0, \sum_{n=1}^{\infty} \alpha_{n}=$ $\infty, \sum_{i=0}^{N} \beta_{i}=1$, and $\lim _{n \rightarrow \infty} r_{n}=\infty$. Then $\left\{x_{n}\right\}$ converges strongly to the minimum-norm element of $\mathcal{F}$.

Remark 4.2 Theorem 3.1 provides convergence scheme to the common minimum-norm zero of a finite family of monotone mappings which improves the results of Bauschke et al. [7] to Banach spaces more general than Hilbert spaces. We also note that our results complement the results of Zegeye and Shahzad [9] and Hu and Liu [10] which are convergence results for accretive mappings.

Competing interests

The authors declare that they have no competing interests.

Authors' contributions

All authors contributed equally to this work. All authors read and approved final manuscript.

Author details

${ }^{1}$ Department of Mathematics, University of Botswana, Pvt. Bag 00704, Gaborone, Botswana. ${ }^{2}$ Department of Mathematics, King Abdulaziz University, P.O. Box 80203, Jeddah, 21589, Saudi Arabia.

\section{Acknowledgements}

This article was funded by the Deanship of Scientific Research (DSR), King Abdulaziz University, Jeddah. The second author acknowledges with thanks DSR for financial support. 
References

1. Byrne, C: Iterative oblique projection onto convex sets and the split feasibility problem. Inverse Probl. 18(2), 441-453 (2002)

2. Censor, Y, Elfving, T: A multiprojection algorithm using Bregman projections in a product space. Numer. Algorithms 8(2-4), 221-239 (1994)

3. Oin, $X$, Kang, Jl, Cho, YJ: On quasi-variational inclusions and asymptotically strict pseudo-contractions. J. Nonlinear Convex Anal. 11, 441-453 (2010)

4. Takahashi, S, Takahashi, W, Toyoda, M: Strong convergence theorems for maximal monotone operators with nonlinear mappings in Hilbert spaces. J. Optim. Theory Appl. 147, 27-41 (2010)

5. Zhang, M: Iterative algorithms for common elements in fixed point sets and zero point sets with applications. Fixed Point Theory Appl. 2012, 21 (2012)

6. Takahashi, W: Nonlinear Functional Analysis. Kindikagaku, Tokyo (1988) (Japanese)

7. Bauschke, $\mathrm{HH}$, Combettes, $\mathrm{PL}$, Reich, S: The asymptotic behavior of the composition of two resolvents. Nonlinear Anal. 60(2), 283-301 (2005)

8. Hundal, H: An alternating projection that does not converge in norm. Nonlinear Anal. 57, 35-61 (2004)

9. Zegeye, $\mathrm{H}$, Shahzad, N: Strong convergence theorems for a common zero of a finite family of $m$-accretive mappings. Nonlinear Anal. 66, 1161-1169 (2007)

10. Hu, L, Liu, L: A new iterative algorithm for common solutions of a finite family of accretive operators. Nonlinear Anal. 70, 2344-2351 (2009)

11. Aoyama, K, Kohsaka, F, Takahashi, W: Proximal point method for monotone operators in Banach spaces. Taiwan J. Math. 15(1), 259-281 (2011)

12. Alber, Y: Metric and generalized projection operators in Banach spaces: properties and applications. In: Kartsatos, AG (ed.) Theory and Applications of Nonlinear Operators of Accretive and Monotone Type. Lecture Notes in Pure and Appl. Math., vol. 178, pp. 15-50. Dekker, New York (1996)

13. Kamimura, S, Kohsaka, F, Takahashi, W: Weak and strong convergence theorems for maximonotone operators in a Banach spaces. Set-Valued Anal. 12, 417-429 (2004)

14. Matsushita, S, Takahashi, W: A strong convergence theorem for relatively nonexpansive mappings in a Banach space. J. Approx. Theory 134, 257-266 (2005)

15. Kamimura, S, Takahashi, W: Strong convergence of proximal-type algorithm in a Banach space. SIAM J. Optim. 13, 938-945 (2002)

16. Zegeye, H, Ofoedu, EU, Shahzad, N: Convergence theorems for equilibrium problem, variational inequality problem and countably infinite relatively quasi-nonexpansive mappings. Appl. Math. Comput. 216, 3439-3449 (2010)

17. $\mathrm{Xu}, \mathrm{HK}$ : Another control condition in an iterative method for nonexpansive mappings. Bull. Aust. Math. Soc. 65 109-113 (2002)

18. Maingé, PE: Strong convergence of projected subgradient methods for nonsmooth and non-strictly convex minimization. Set-Valued Anal. 16, 89-912 (2008)

19. Rockafellar, RT: Monotone operators and proximal point algorithm. Trans. Am. Math. Soc. 194, 75-88 (1970)

10.1186/1029-242X-2013-566

Cite this article as: Zegeye and Shahzad: An algorithm for a common minimum-norm zero of a finite family of monotone mappings in Banach spaces. Journal of Inequalities and Applications 2013, 2013:566

\section{Submit your manuscript to a SpringerOpen ${ }^{\circ}$ journal and benefit from:}

- Convenient online submission

Rigorous peer review

- Immediate publication on acceptance

Open access: articles freely available online

- High visibility within the field

- Retaining the copyright to your article 